\title{
Joint Access Point Placement and Channel Assignment for 802.11 Wireless LANs
}

\author{
Xiang Ling \\ School of Communication and Information Engineering \\ University of Electronic Science and Technology of China \\ Chengdu, China \\ xiangling@uestc.edu.cn
}

\author{
Kwan Lawrence Yeung \\ Dept of Electrical and Electronic Engineering \\ University of Hong Kong \\ Hong Kong, China \\ kyeung@eee.hku.hk
}

\begin{abstract}
To deploy a multi-cell 802.11 wireless local area network (WLAN), access point (AP) placement and channel assignment are two primary design issues. For a given pattern of traffic demands, we aim at maximizing not only the overall system throughput, but also the fairness in resource sharing among mobile terminals. A novel method for estimating the system throughput of multi-cell WLAN is proposed. An important feature of this method is that co-channel overlapping is allowed. Unlike conventional approaches that decouple AP placement and channel assignment into two phases, we propose to jointly solve the two problems for better performance. Due to the high computational complexity involved in exhaustive searching, an efficient local searching algorithm, called patching algorithm, is also designed. Numerical results show that for a typical indoor environment, patching algorithm can provide a close-to-optimal performance with much lower time complexity.
\end{abstract}

Keywords-wireless local area networks; 802.11; access point placement; channel assignment

\section{INTRODUCTION}

The wireless local area network (WLAN) has been an astounding success since IEEE 802.11 standards have been published to rule the development of WLAN. As more and more multi-cell 802.11 WLANs are being deployed to provide seamless coverage, a systematic approach for determining where an access point (AP) should be placed, and which channel an AP should be assigned become increasingly important.

Efforts dedicated to WLANs design have produced some useful results. Rodrigues [1] reported a real experience of AP placement in an indoor environment which aims at maximizing the total receiving signal strength. Lee [2] considered load balancing among multiple APs by minimizing the load carried by the heaviest-loaded AP. However, these two approaches can give no solution when co-channel interference between cells exists. Kamenetsky [3] combined pruning and other refining algorithms to solve AP placement in an ad-hoc network. Leung [4] discussed the frequency assignment for multi-cell WLAN while assuming an ideal hexagonal coverage for each AP. Park [5] introduced the throughput measurements in an office and a conference room. Hills [6] described the general procedure in a large-scale WLAN design, in which AP placement and channel assignment are carried out in two separated phases.
In this paper, we want to jointly solve the two problems, AP placement and channel assignment, by a single algorithm, with a new objective of simultaneously maximizing the total system throughput and maintaining fair resource sharing. To evaluate and compare different design solutions, an original method for throughput estimation is first proposed. Unlike existing approaches, our estimation method allows co-channel overlapping between cells. In order not to rely on the brutal force for finding the global optimal solution, a heuristic called patching algorithm is also proposed.

\section{802.11 SPECIFICATION AND DESSIGN PROCESS}

Among the IEEE 802.11 standard series, $802.11 \mathrm{~b}$ is the most-widely deployed version. It uses direct sequence spread spectrum (DSSS) as physical layer and adopts carrier sense multiple access with collision avoidance (CSMA/CA) in MAC layer. The data rates supported are 1, 2, 5.5 and $11 \mathrm{Mbps}$ depending on the received signal sensitivity thresholds (RXThresh). If the received signal strength is below RXThresh but above carrier sense threshold (CSThresh), carrier busy can be detected. In an $802.11 \mathrm{~b}$ network, there are 13 channels regulated by ETSI or 11 channels regulated by FCC. Adjacent channels have spectrum overlapping; so generally only 3 fully isolated frequency bands can be assigned for eliminating adjacent channel spectral interference. To avoid collision caused by hidden terminals, Request-to-Send (RTS) / Clear-toSend (CTS) mechanism is usually adopted.

The target of WLAN design is to satisfy the traffic demands from the mobile terminals while keeping the deployment cost low. A generic design process for deploying a multi-cell WLAN system consists of four phases $[1,2]$ :

1) Partitioning the service area into grids. Each grid is the basic spot for terminal counting and signal strength measuring.

2) Choosing candidate locations for APs.

3) Signal strength measurement. The measurement should be taken at every covered grid for every AP candidate. The signal strength could also be predicted by analysis [7].

4) Deciding AP placement and channel assignment. Our research in this paper focuses on this phase.

\section{THROUGHPUT ESTIMATION}

To jointly carry out AP placement and channel assignment, we must have a simple and efficient way to evaluate and 
compare different solutions. In this section we propose an efficient method for throughput estimation of a multi-cell WLAN system.

Reference [8] analyzed the system performance of one AP's service region (i.e. a single cell), from which the probability $\left(P_{t r}\right)$ that at least one mobile terminal (MT) transmits the packet in the considered slot time, and the probability $\left(P_{s}\right)$ that a transmission seizes the channel successfully are derived as follows:

$$
\begin{gathered}
P_{t r}=1-(1-\tau)^{n} \\
P_{s}=\frac{n \tau(1-\tau)^{n-1}}{P_{t r}}
\end{gathered}
$$

where $n$ is the total number of mobile terminals in a cell; $\tau$ is the probability that a mobile terminal's backoff timer decreases to zero (and a packet will be sent). Please refer to [8] for more details.

There are three scenarios of channel conditions shown in Fig. 1: (a) channel idle; (b) channel held by a successful transmission; and (c) channel collision, i.e. more than one MT transmit at the same time. The corresponding channel idle probability $P_{\text {idle }}$, successful transmission probability $P_{\text {succ }}$, and the channel collision probability $P_{\text {coll }}$ can be obtained as follows.

$$
\begin{gathered}
P_{\text {idle }}=1-P_{t r} \\
P_{\text {succ }}=P_{t r} P_{s} \\
P_{\text {coll }}=P_{t r}\left(1-P_{s}\right)
\end{gathered}
$$

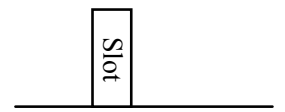

(a) Channel idle

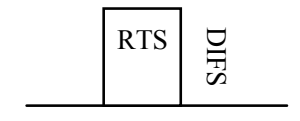

(c) Channel collision

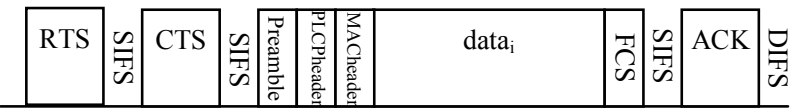

(b) Channel held by a successful transmission

Figure 1. Three scenarios of channel conditions

Based on $802.11 \mathrm{~b}$ specification, let the channel idle duration time $T_{\text {idle }}$ in Fig. 1a be $20 \mu$ s. In Fig. 1b, if RTS/CTS mechanism is adopted, the total channel holding time for mobile terminal $\mathrm{MT}_{\mathrm{i}}$ 's packet transmission, $T_{i}$, is given by:

$$
\begin{gathered}
T_{i}=T_{R T S}+T_{C T S}+T_{\text {preamble }}+T_{M P D U-i}+T_{A C K}+T_{D I F S}+3 T_{S I F S} \\
T_{M P D U-i}=\frac{L_{P L C \text { Cheader }}}{1 M b p s}+\frac{L_{M A C h e a d e r}+L_{\text {data }-i}+L_{F C S}}{\text { DataRate }_{i}}
\end{gathered}
$$

where $T_{D I F S}, T_{S I F S}$ are the corresponding interframe spaces; $T_{R T S}, T_{C T S}, T_{A C K}$ are time for each handshaking message; $T_{\text {preamble }}$ is the time consumed by preamble; $T_{M P D U-i}$ is the total transmission time for $\mathrm{MT}_{\mathrm{i}}$ 's data packet; $L_{P L C \text { Pheader }}, L_{\text {MACheader }}$, $L_{F C S}$ are the sizes of the corresponding packet header and trailer fields; $L_{\text {data-i }}$ is the size of the payload; DateRate ${ }_{i}$ is $11,5.5,2$ or $1 \mathrm{Mbps}$ depending on $\mathrm{MT}_{\mathrm{i}}$ 's received signal strength.

When channel collision occurs, the sender will not receive CTS frame and the collision can be quickly detected; see Fig.1c. So the collision time $T_{\text {coll }}$ is:

$$
T_{\text {coll }}=T_{\text {RTS }}+T_{D I F S}
$$

If constant MAC packet size is assumed, low data rate terminals will hold the channel longer than high data rate terminals [9]. For example, if CBR service is carried by 1500byte UDP packet, the channel holding time for sending a UDP packet using $1 / 2 / 5.5 \mathrm{Mbps}$ data rate are 5.80/3.16/1.48 times of that using $11 \mathrm{Mbps}$.

To cover the whole building or campus by multi-cell WLAN, some cells maybe be assigned to use the same channel. The relationship between such co-channel cells falls into three types:

1) Overlapped cells. In Fig. 2a, shaded areas denote the receiving range (where signal strength $>$ RXThresh), and the dashed circles denote the interference range (where signal strength $>$ CSThresh). $\mathrm{MT}_{1}$ can sense the carrier from both $\mathrm{AP}_{1}$ and $\mathrm{AP}_{2}$.

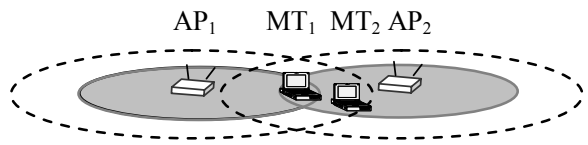

(a) Overlapped cells

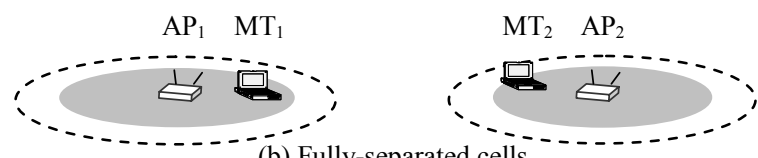

(b) Fully-separated cells

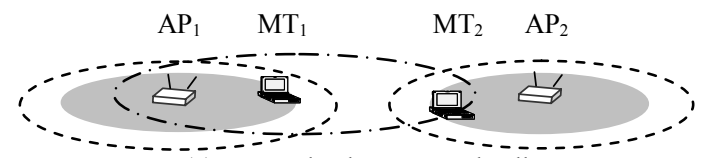

(c) Incompletely-separated cells

Figure 2. Relationship of AP cells. (The regular shaped cell is for illustration purpose only.)

2) Fully-separated cells. In Fig. 2b, the distance between two cells is far enough, such that the associated MTs in one cell cannot interfere the data transmission in the adjacent cell.

3) Incompletely-separated cells. In Fig. 2c, two cells are separated but the distance between them is not far enough: $\mathrm{MT}_{1}$ located at the boundary of cell1 can receive the co-channel signals from $\mathrm{MT}_{2}$ who is associated with $\mathrm{AP}_{2}$. In this case, $\mathrm{MT}_{1}$ cannot send (to $\mathrm{AP}_{1}$ ) simultaneously as $\mathrm{MT}_{2}$. They have mutual interference. It should be noted that this case of incompletelyseparated cells has been ignored in $[1,2,4]$. 
Both contention within a cell and the co-channel interference from adjacent cells can restrain a MT from data transmission. Assume $\mathrm{MT}_{1}$ is associated to $\mathrm{AP}_{1}$, the four possible coverage scenarios are shown in Fig. 3. For each scenario, the area that a potential restrainer of $\mathrm{MT}_{1}$ may reside is shaded. (The solid and dashed circles delineate the boundaries of AP's receiving and interference ranges respectively.)

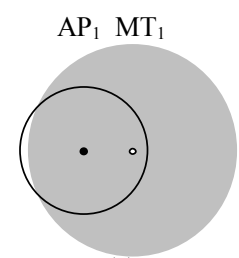

(a)

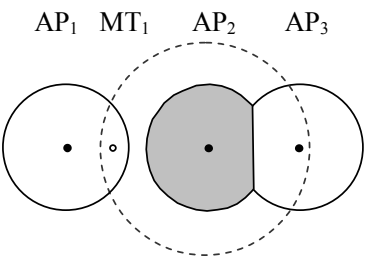

(c)

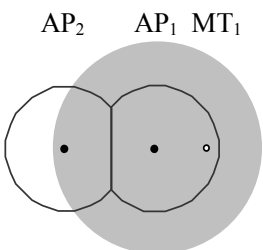

(b)

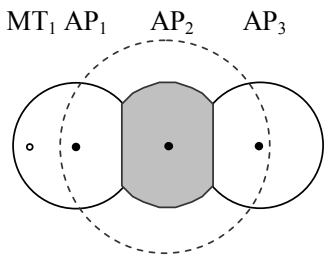

(d)
Figure 3. Restrain criteria

From Fig. 3, the potential restrainers of $\mathrm{MT}_{1}$ can be found:

1) Mobile terminals in $M T_{1}$ 's radiating range. The shaded area in Fig. 3a illustrates where the mobile terminals can be heard by $\mathrm{MT}_{1}$ directly.

2) Mobile terminals located in $A P_{1}$ 's interference range. Refer to Fig. 3b.

3) If $M T_{1}$ is in $A P_{2}$ 's interference range, mobile terminals associated to $A P_{2}$ will restrain $M T_{1}$. In Fig. $3 \mathrm{c}$, when $\mathrm{AP}_{2}$ communicates with its subscribers, $\mathrm{MT}_{1}$ detects the carrier busy signal. $\mathrm{MT}_{1}$ will keep silence until transmission in $\mathrm{AP}_{2}$ 's service area ends.

4) If $A P_{1}$ and $A P_{2}$ are so close that they can interfere with each other directly, mobile terminals associated to $\mathrm{AP}_{2}$ are $M T_{1}$ 's restrainers. This is shown in Fig. $3 \mathrm{~d}$, where $\mathrm{AP}_{1}$ detects transmission in $\mathrm{AP}_{2}$ 's service area and then keeps silence. When $\mathrm{MT}_{1}$ requests transmission to $\mathrm{AP}_{1}, \mathrm{AP}_{1}$ will not respond.

Based on the potential restrainers identified above, $\mathrm{MT}_{\mathrm{i}}$ 's throughput $T H R_{i}$ can be found:

$$
\begin{gathered}
\text { THR }_{i}=\text { DataRate }_{i} \times \operatorname{Pr}_{i} \times \text { Efficiency }_{i} \\
\operatorname{Pr}_{i}=\frac{\frac{P_{\text {succ }}}{1+\sum_{j} r s n_{i j}} T_{i}}{\frac{P_{\text {succ }}}{1+\sum_{j} r s n_{i j}}\left[T_{i}+\sum_{j}\left(r s n_{i j} * T_{j}\right)\right]+P_{\text {idle }} * T_{\text {slot }}+P_{\text {coll }} * T_{\text {coll }}} \\
\text { Efficiency }_{i}=\frac{L_{\text {data }-i} / \text { DataRate }_{i}}{T_{i}}
\end{gathered}
$$

where $\operatorname{Pr}_{i}$ is the probability that $\mathrm{MT}_{\mathrm{i}}$ holds the channel. $R s n_{i j}$ equals to 1 if $\mathrm{MT}_{\mathrm{j}}$ is a restrainer of $\mathrm{MT}_{\mathrm{i}}$; otherwise it equals to 0 . Assume each MT always has sustained traffic to send (i.e. every MT will request channel if channel idle is detected). Note that more restrainers make it harder for $\mathrm{MT}_{\mathrm{i}}$ to seize the channel. This can be seen from (10). Efficiency Ef $_{i}$ (11) is the portion of time used by $\mathrm{MT}_{\mathrm{i}}$ for transmitting its payload.

The total system throughput $T H R_{\text {total }}$ can be estimated by adding the respective throughput of individual MTs.

$$
T H R_{\text {total }}=\sum_{i} T H R_{i}
$$

\section{NEW OBJECTIVE FUNCTION}

Several objective functions (OF) for AP placement and channel assignment were proposed in the literatures. Pathloss minimization was proposed in [3]. Although AP placement can be determined by pathloss, it cannot be used for solving the channel assignment problem. In this case, AP placement and channel assignment must be split into two separated phases. Load balance was chosen as the objective function in [2], and the resulting algorithm cannot solve the channel assignment problem effectively. In fact, prohibiting co-channel overlapping is the only constraint for channel assignment in [2]. If more and more APs are placed for achieving higher system throughput, co-channel overlapping occurs inevitably. If this happens, the algorithm in [2] cannot return feasible solution.

In this section, we design a new objective function for joint optimization of AP placement and channel assignment. This function, shown in (13), aims at optimizing both the system throughput and the fairness among mobile terminals. It is a product between $T H R_{\text {total }}$ from (12) and the fairness index $\beta$ from (14). (Note that AP placement and channel assignment are carried out in the system design phase; a detailed throughput analysis based on the actual traffic generation is not needed. And the sustained traffic demand represents the worst situation that system will encounter.) In (14), $\beta$ is defined as a measure of the deviations of the throughputs acquired by individual MTs.

$$
\begin{array}{r}
O F=T H R_{\text {total }} \times \beta \\
\beta=\frac{\left(\sum_{i=1}^{N} T H R_{i}\right)^{2}}{N \times \sum_{i=1}^{N} T H R_{i}^{2}}
\end{array}
$$

$N$ is the total number of MTs in the system. The fairness index $\beta$ approaches 1 when all MTs have exactly the same throughput. When the throughputs are heavily unbalanced, $\beta$ converges to $1 / N$.

\section{PATCHING AlgORITHM}

Based on the objective function in (13), an optimal solution for joint AP placement and channel assignment can be found by exhaustive searching. If the number of AP candidates is 
large, the exhaustive search becomes extremely complex. Assume $M$ APs should be chosen from $L$ candidates and three isolated channels are available for channel assignment. The number of possible solutions is on the order of $C_{L}^{M}\left(3^{M-1}+1\right) / 2$. Brutal force searching is thus not feasible. In this section, we propose a time-efficient local searching heuristic, called patching algorithm.

Patching algorithm places APs one by one to cover the traffic demands until a pre-defined number of APs are placed. At each step, patching algorithm attempts to select one AP from the remaining candidate pool, which can provide the largest $\mathrm{OF}$ value together with those already placed APs. The pseudo-code for patching algorithm is outlined in Table I.

TABLE I. Patching Algorithm Psedo-Code

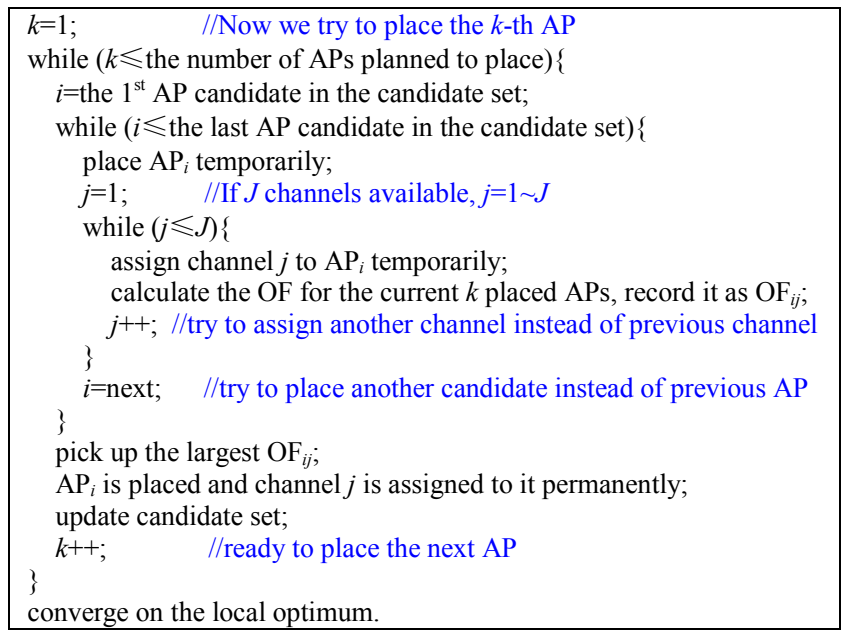

The patching algorithm starts with an initial candidate set that contains all candidate $\mathrm{APs}$, i.e. $\left\{\mathrm{AP}_{1}, \mathrm{AP}_{2}, \ldots \mathrm{AP}_{L}\right\}$. Then the candidate that gives the largest $\mathrm{OF}$ value is selected for first AP placement. The AP candidate set is updated by deleting the placed AP. To select and place the $2^{\text {nd }}$ AP, we consider the remaining $L-1$ candidate APs together with three possible channel assignments each. The candidate AP with a suitable channel that gives the largest $\mathrm{OF}$ value among all possible combinations is then selected for the second AP placement and channel assignment. This process is repeated until a predetermined number of APs are placed. In each iteration, newly placed AP may cause some mobile terminals to re-associate with it, and the restrain range of some mobile terminals will also be affected. If this happens, the throughput of those affected mobile terminals should be re-estimated using (9).

\section{PERFormance EVAlUATIONS}

\section{A. Throughput Estimation Verification}

First a simple scenario of two overlapped cells (or APs) is simulated by NS-2 to verify the throughput estimation method we proposed in Section III. The network topology includes two APs; MTs are uniformly distributed and communicate with the fixed network via these two APs. UDP connections are established from the MTs to the fixed network for carrying 1500-byte CBR traffic. Let the wireless interface be the bottleneck in the transmission. The transmission power is set to $20 \mathrm{dBm}$. The RXThreshs are $-75 /-79 /-81 /-84 \mathrm{dBm}$ for different data rate receivers, and CSThresh is set to $-94 \mathrm{dBm}$.

If a same channel is assigned to these two APs, the estimated throughputs and those obtained by simulations are compared in Fig. 4. The $\mathrm{X}$-axis is the distance between the two APs. When Distance $=0 \mathrm{~m}$ (i.e. these two APs are fully superposed), there are 13/8/4/12 mobile terminals working at $11 / 5.5 / 2 / 1 \mathrm{Mbps}$ data rates respectively, so the system throughput is given by

$$
\begin{aligned}
& \text { THR }_{\text {total }}=13 \times 11 \mathrm{Mbps} \times 0.00908 \times 0.469+8 \times 5.5 \mathrm{Mbps} \times 0.0134 \times 0.634 \\
& +4 \times 2 \mathrm{Mbps} \times 0.0287 \times 0.817+12 \times 1 \mathrm{Mbps} \times 0.0526 \times 0.890=1.73 \mathrm{Mbps}
\end{aligned}
$$

From the equation above, we can also see that the channel holding probability for each MT with $11 \mathrm{Mbps}$ data rate is 0.00908; and their throughput efficiency is 0.469 . Note that for Distance $=0 \mathrm{~m}$, all MTs have the same restrain range.

It is evident from Fig. 4 that the system throughput is poor when the distance between APs is less than the interference radius $R_{C S}(\approx 1062 \mathrm{~m})$. In this case, the two co-channel APs cannot be fully utilized. If the distance is longer than $R_{C S}$, both APs work with full utilization, so the system throughput is doubled to give $3.47 \mathrm{Mbps}$.

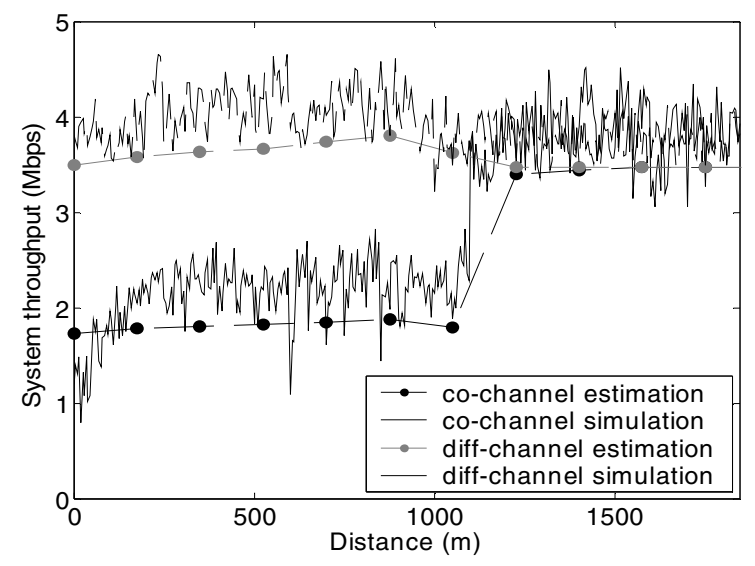

Figure 4. Total co-channel and diff-channel throughput according to the distance between two APs

Fig. 4 also compares the scenario that different channels are assigned to the two APs, denoted by "diff-channel". We can see that the diff-channel overlapping between cells can slightly improve the system throughput. This is because mobile terminals residing at the boundary of $\mathrm{AP}_{1}$ can only work at low data rate if they associate to $\mathrm{AP}_{1}$; when overlaps with another $\mathrm{AP}_{2}$ of a different channel, some low data rate terminals can now re-associate to $\mathrm{AP}_{2}$; then those terminals can get a higher data rate. Meanwhile, the overlapping between diff-channel cells will not cause interference.

To conclude, the above study shows that our throughput estimation method can provide a satisfactory prediction with or without co-channel overlapping. The results in the subsequent subsections are thus based on this throughput estimation. 


\section{B. Environmental Assumption}

Following the design process described in Section II, the service area partitioning for a typical building floor is shown in Fig. 5, with a gird granularity of $5 \mathrm{~m}$. A non-uniform distribution of 0 to 6 MTs per gird is illustrated by different colors (or grey levels). The hotspots, where the MT density is substantially higher can be found. The 16 AP candidate locations are given and numbered from $0 \sim 15$.

The signal strength is predicted by Two-Ray-Ground model [12]. The pathloss values of signal traveling across concrete wall and turning right angle at the corner are $15 \mathrm{~dB}$ and $10 \mathrm{~dB}$. The predicted receiving signal strengths in $\mathrm{dBm}$ for candidate $\mathrm{AP}_{2}$ are shown in Fig. 5 as an example.

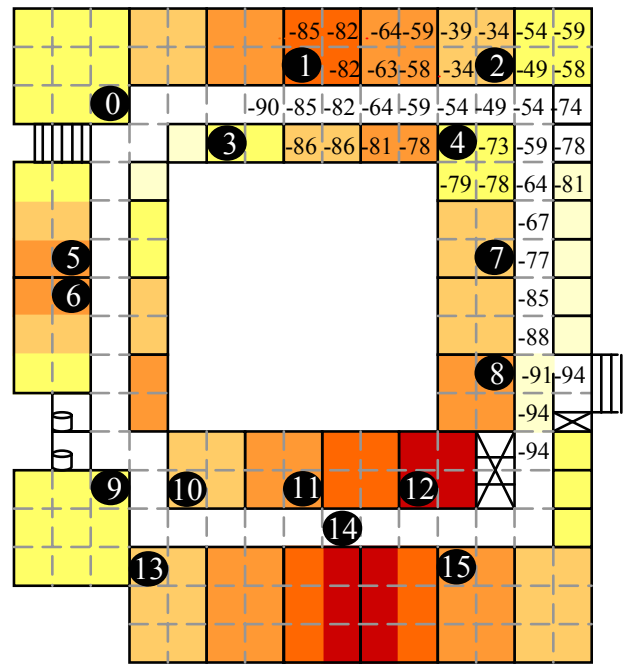

Figure 5. MT distribution and candidate AP locations in a building

\section{Objective Functions and Global Optimums}

For convenience, let the objective function defined in (13) be $\mathrm{OF}_{1}$, the objective function proposed in [2] be $\mathrm{OF}_{2}$, and the objective function adopted in [1] be $\mathrm{OF}_{3}$. $\mathrm{OF}_{2}$ attempts to balance the loads among APs. $\mathrm{OF}_{3}$ tries to maximize the sum of received signal strengths.

Fig. 6 compares the optimal solutions obtained by exhaustive searching based on $\mathrm{OF}_{1}, \mathrm{OF}_{2}$ and $\mathrm{OF}_{3}$. We can see that our joint AP placement and channel assignment based on $\mathrm{OF}_{1}$ provides the highest system throughput and close-to-best fairness index. Note that more than one optimal solution can be found based on $\mathrm{OF}_{2}$ and $\mathrm{OF}_{3}$. Their distributions are illustrated and their average values are connected by dashed lines. From Fig. 6, we can see that there is no solution for $\mathrm{OF}_{2}$ when less than 6 APs are placed. This is because optimization method in [2] has a constraint that every MT must be served. If less than 6 APs are placed, no solution can be found to cover all terminals. Besides, when more than 8 APs are placed, there is no solution for both $\mathrm{OF}_{2}$ and $\mathrm{OF}_{3}$. This is because those placement algorithms do not allow co-channel overlapping. If more than 8 APs are placed, co-channel interference between APs cannot be avoided.

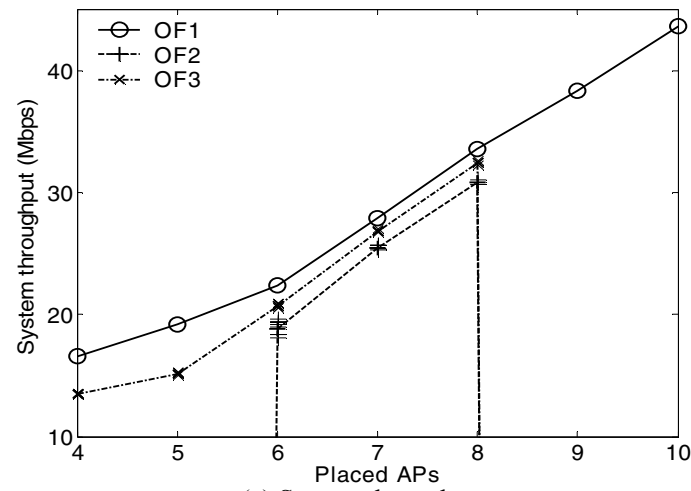

(a) System throughput

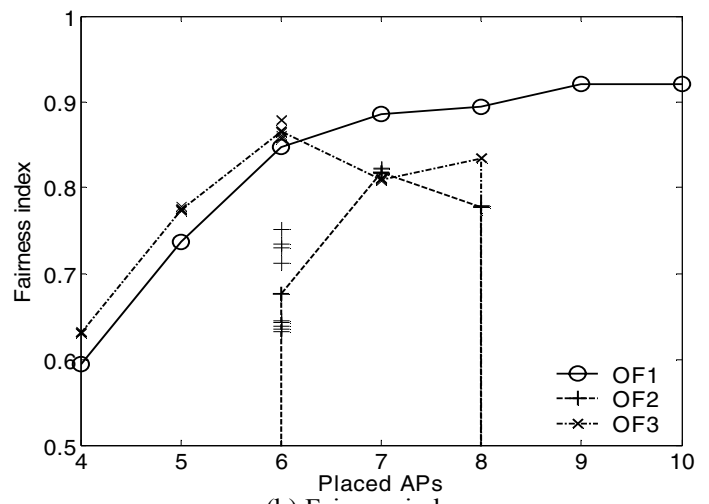

(b) Fairness index

Figure 6. System throughput and fairness index of optimums found by exhaustive search based on three objective functions

\section{Patching Algorithm and Local Optimums}

The patching algorithm described in Section $\mathrm{V}$ can significantly reduce the computational complexity. For solving the same problem as that in the previous subsection, Table II summarizes the number of solutions that are visited by the exhaustive searching and the patching algorithm respectively.

\section{TABLE II. COMPUTATIONAL COMPLEXITY}

\begin{tabular}{|l|l|l|}
\hline $\begin{array}{c}\text { Visited } \\
\text { solutions }\end{array}$ & $\begin{array}{c}\text { Exhaustive } \\
\text { searching }\end{array}$ & $\begin{array}{c}\text { Patching } \\
\text { algorithm }\end{array}$ \\
\hline 4 & 25480 & 127 \\
\hline 5 & 179088 & 163 \\
\hline 6 & 976976 & 193 \\
\hline 7 & 4175600 & 226 \\
\hline 8 & 14079780 & 253 \\
\hline 9 & 16777216 & 277 \\
\hline 10 & 16777216 & 298 \\
\hline
\end{tabular}

The OF values found by the exhaustive searching and the patching algorithm are compared in Fig. 7. We can see that the performance of patching algorithm is very close to the optimal.

We next compare the patching algorithm with another algorithm Prun+RCC in Fig. 8. Prun+RCC spits AP placement and channel assignment into two separated phases. The pruning algorithm (Prun) is adopted in AP placement phase for minimizing pathloss [3], and a random channel convergence (RCC) algorithm is used in the channel assignment phase [4]. $\mathrm{RCC}$ adjusts the channel assignment of the bottleneck AP's 


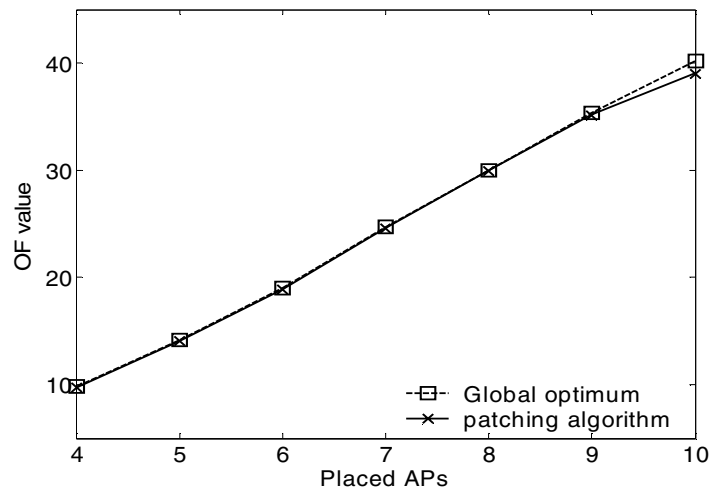

Figure 7. OF values got by patching algorithm and exhaustive searching

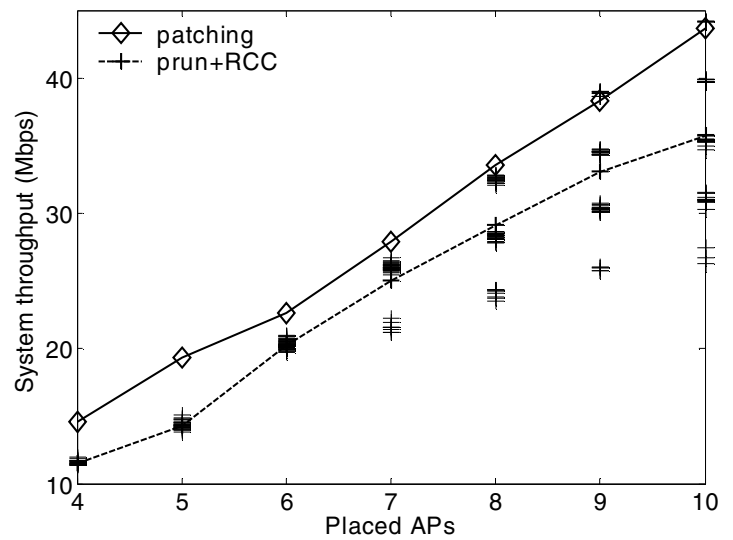

(a) System throughput

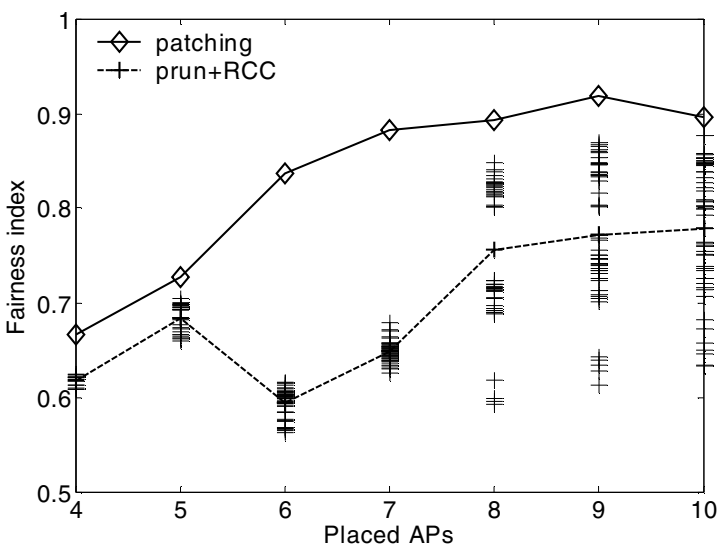

(b) Fairness index

Figure 8. System throughput and fairness index of local optimums

neighbors to minimize its traffic load. From Fig. 8, we can see that more than one solutions $(>20)$ can be found for Prun $+\mathrm{RCC}$ algorithm. This is because the RCC algorithm only adjusts the channel assignment in bottleneck AP's neighbors. For a heavily unbalanced mobile terminal distribution, the bottleneck AP usually locates at the hotspot area. Adjusting the channels in its neighboring APs cannot reduce the load of the bottleneck AP effectively. If the bottleneck cannot be removed from the hotspot to other "cooler" spots, this algorithm cannot optimize the channel assignment in light traffic demand areas. As such, channels can be assigned to the APs in light traffic demand areas freely; this generates many local optimal solutions. Overall speaking, Fig. 8 shows that splitting AP placement and channel assignment into two phases leads to poor performance than patching algorithm.

\section{CONCLUSIONS}

In this paper, we first proposed an original method to estimate the throughput of a multi-cell 802.11b WLAN system. The co-channel overlapping between cells is allowed. Then an optimization method for joint AP placement and channel assignment was proposed, which aims at maximizing both the system throughput and the fairness index. Its optimal solutions can be found by exhaustive searching. To reduce the computational complexity, a simple local searching heuristic, called patching algorithm, was designed and analyzed. We showed that in a typical indoor environment, patching algorithm provides close-to-optimal system throughput and fairness index.

\section{REFERENCES}

[1] R.C.Rodrigues, G.R.Mateus, and A.A.F.Loureiro, "On the design and capacity planning of a wireless local area network," IEEE/IFIP Network Operations and Management Symposium 2000.

[2] Y.Lee, K.Kim, and Y.Choi, "Optimization of AP placement and channel assignment in wireless LANs," Proceedings of the $27^{\text {th }}$ Annual IEEE Conference on Local Computer Networks, 2002.

[3] M.Kamenetsky and M.Unbehaun, "Coverage planning for outdoor wireless LAN systems," International Zurich Seminar on Broadband Communications, 2002.

[4] K.K.Leung and B-J."J"Kim, "Frequency assignment for multi-cell IEEE 802.11 wireless networks," VTC'03 Fall.

[5] J.A.Park, S.K.Park, P.D.Cho, and K.R.Cho, "Analysis of spectrum channel assignment for IEEE $802.11 \mathrm{~b}$ wireless LAN," $5^{\text {th }}$ International Symposium on Wireless Personal Multimedia Communications, 2002.

[6] A.Hills, "Large-scale wireless LAN design," IEEE Communications Magazine, vol. 39, no. 11, pp. 98-107, Nov 2001.

[7] S.Armour, A.Doufexi, B-S.Lee, A.Nix, and D.Bull. "The impact of power limitations and adjacent residence interference on the performance of WLANs for home networking applications," IEEE Trans on Consumer Electronics. vol. 47, no.3, pp. 502 511, August 2001.

[8] G.Bianchi, "Performance analysis of IEEE 802.11 distributed coordination function," IEEE Journal on Selected Areas in Communications, vol. 18, no. 3, pp. 535 547, March 2000.

[9] M.Heusse, F.Rousseau, G.Berger-Sabbatel, and A.Duda, "Performance anomaly of $802.11 \mathrm{~b}$," Infocom 2003 .

[10] F.Cali, M.Conti, and E.Gregori. "IEEE 802.11 wireless LAN: capacity analysis and protocol enhancement," Infocom'98, 1998.

[11] Y.Xiao and J.Rosdahl. "Throughput and delay limits of IEEE 802.11," IEEE Communications Letters. vol. 6, no. 8, August 2002.

[12] www.isi.edu/nsnam/ns/node217.html 